\title{
Optical Signals from Neurons with Internally Applied Voltage- Sensitive Dyes
}

\author{
Srdjan Antić and Dejan Zečević \\ Institute for Biological Research, Belgrade, Yugoslavia
}

\begin{abstract}
We carried out experiments to monitor optically the generation and spread of action potentials and subthreshold potentials in the processes of individual neurons in ganglia of the snail, Helix aspersa. The neurons were selectively stained by intracellular pressure injection of voltage-sensitive dyes. Optical signals were detected by a system for fast, multiple-site optical monitoring, utilizing a silicon photodiode array.
\end{abstract}

After testing 30 voltage-sensitive dyes using absorption, we concluded that this mode was probably not sensitive enough to allow monitoring neuronal signals from distal processes. Satisfactory signals were obtained in fluorescence measurements using a newly synthesized styryl dye, JPW1114, specifically designed for intracellular application. There was an improvement in sensitivity (as defined by the signal-to-noise ratio) by a factor of about 50 over previously reported absorption and fluorescence signals from neuronal processes stained by either intra- or extracellular application of dyes.

Recordings with good signal-to-noise ratio and adequate spatial and temporal resolution were obtained simultaneously from the cell body and long axonal branches. From this data, the site of action potential initiation was determined. Also, the propagation velocity of the action potential was calculated for different axonal segments; the results suggest that different regions have different velocities ranging from $0.53 \mathrm{~m} / \mathrm{sec}$ to $0.07 \mathrm{~m} / \mathrm{sec}$. The present sensitivity was adequate to allow the recording of a 10 $\mathrm{mV}$ hyperpolarizing electrotonic response along axonal branches and to observe directly the decline of this passive response with distance from the site of stimulation. Relatively modest improvements in sensitivity will allow systematic analyses of the spread and summation of synaptic potentials in individual neurons.

[Key words: voltage-sensitive dyes, intracellular staining, optical signals, fluorescence, molluscan neurons, neurites]

In both classical (Kandel et al., 1967) and more recent (e.g., Cleary and Byrne, 1993) work on functional neuronal networks, neurons were represented as isopotential elements that perform

Received Apr: 18, 1994; revised June 10, 1994; accepted Aug. 12, 1994.

This work was supported in part by Grants NS 28433 and NS 08437 from NINDS. We thank Lawrence B. Cohen for the help with the optical recording apparatus. We are grateful to Amiram Grinvald, Rina Hildesheim, Leslie Loew, and Joe Wuskel for kindly providing dyes and to Lawrence Cohen, William Ross, and Brian Salzberg for helpful comments on the manuscript

Correspondence should be addressed to Dejan Zečević, Department of Cellular and Molecular Physiology, Yale University School of Medicine, $333 \mathrm{Ce}$ dar Street, New IIaven, CT 06510.

Copyright (C) 1995 Society for Neuroscience $0270-6474 / 95 / 151392-14 \$ 05.00 / 0$ simple operations; they either excite or inhibit other elements in the circuit diagram and the function of the circuit depends on the interaction between neurons rather than on the detailed properties of the individual nerve cells.

However, the combined results from modeling studies (e.g., Segev et al., 1992), from studies of the relationship between structure and function for individual neurons (Jacobs and Miller, 1988), and from direct electrical (Regehr et al., 1992, 1993; Stuart and Sakmann, 1994) and optical (Tank et al., 1988; Ross et al., 1993) measurements of electrical properties of neuronal processes, clearly indicate that it may not be sufficient to model real neurons as simple isopotential units. These studies show that the geometry and regional electrical properties of neurons determine how synaptic inputs are integrated into an output signal. While this is true for neurons with passive dendrites, it is especially the case for cells with dendritic processes capable of generating active responses. Recent experimental data show that some dendrites do not behave as passive elements but generate active responses (Llinas and Sugimori, 1980; Tank et al., 1988; Lasser-Ross et al., 1991; Miyakawa et al., 1992; Kim and Connors, 1993; Midtgaard et al., 1993; Regehr et al., 1993; Stuart and Sakmann, 1994). Regehr et al. $(1992,1993)$ suggested that under certain conditions the primary spike initiating zone may be in the dendrites rather than in the initial segment of the axon. However, this has not yet been directly recorded, and the location of the trigger zone remains uncertain.

Although the precise role of regional specializations in excitability of neuronal processes is largely unknown, experimental evidence in several preparations has shown that regional characteristics of neurons may be utilized to provide particular functions. Jacobs and Miller $(1985,1988)$ demonstrated, using laser inactivation techniques, that the directional sensitivity of the cricket to the wind depends precisely on the mapping of sensory information from primary afferents onto the dendritic branching structure of an interneuron. In experiments on motion-sensitive interneurons of the fly, Borst and Egelhaaf (1992) demonstrated that the function of these cells is based on selective activation of restricted parts of dendritic tree by different visual stimuli. Indirect experiments suggested that dendrodendritic interactions occur between motion sensitive interneurons in the fly (Egelhaaf et al., 1993). Also, there is substantial evidence from other preparations that some parts of a dendritic arborization may function as independent units (for review, see Cohen and Wu, 1990).

While it is clear that understanding the functional role of neurons requires determination of regional factors that control electroresponsiveness, progress is restricted because this type of analysis is technically difficult. Adequate experimental techniques for examining the electrical behavior of neuronal pro- 


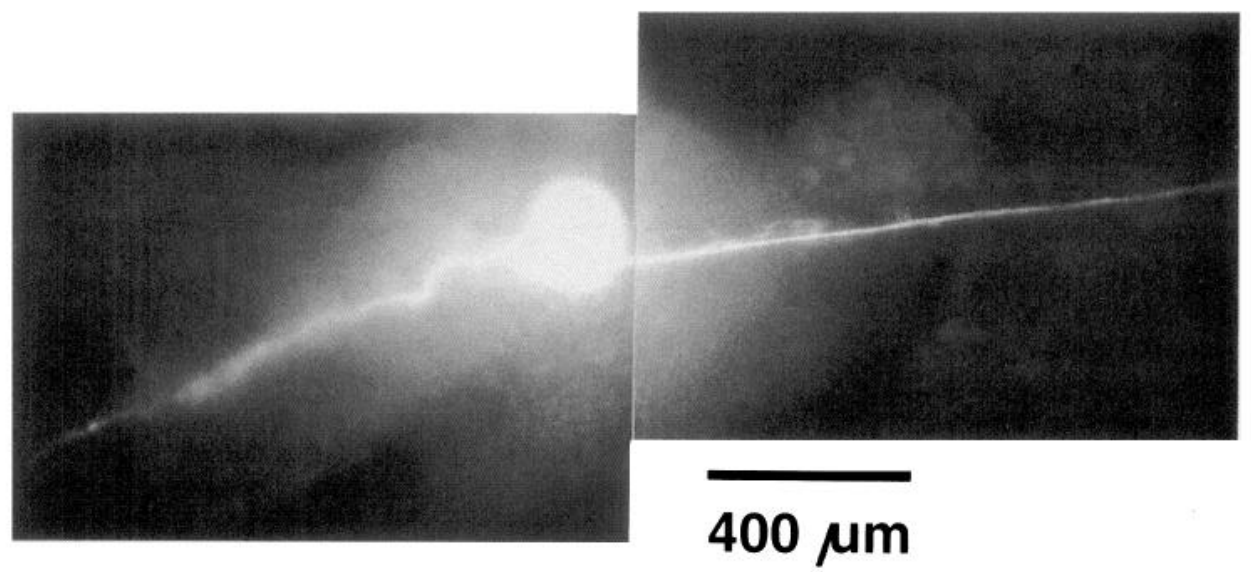

Figure 1. Giant metacerebral interneuron following $12 \mathrm{hr}$ incubation at $15^{\circ} \mathrm{C}$ after injection with the fluorescent voltage-sensitive dye, JPW1114. The cell body and main processes are on the surface of the ganglion and clearly visible in the unfixed preparation. Excitation, $540 \pm 30 \mathrm{~nm}$; dichroic mirror, $570 \mathrm{~nm}$; barrier filter, 610 $\mathrm{nm}$.

cesses are not available. It would be useful if membrane potential transients could be recorded from many different locations on neuronal processes. Obviously, multisite electrical measurements using microelectrodes are not possible because neuronal processes are hard to visualize and too small to tolerate microelectrodes at multiple sites. Direct evidence about electrical signaling in neuronal processes might be achieved by monitoring membrane potential transients at many sites along the dendritic arborization, soma, and axon, by using voltage-sensitive dyes. In 1984, Ross and Krauthamer demonstrated the possibilities and limitations of using extracellular staining and absorption measurements. The work of Obaid et al. (1982) and Grinvald et al. (1987) demonstrated advantages (and difficulties) in using intracellular fluorescent probes. In these experiments, recording of membrane potential transients was possible only under favorable circumstances. It was clear that additional improvements in sensitivity was required before regional characteristics in single neurons could be systematically analyzed.

We report here the results of optical monitoring of the generation and spread of action potentials and subthreshold signals from neurons within intact ganglia with a significant improvement in sensitivity. The obtained sensitivity depends on using fluorescence measurements and selective staining of only one neuron within a ganglion by intracellular injection of membrane impermeant voltage-sensitive dye (Grinvald et al., 1987), and on finding a dye that gives relatively large signals when applied internally.

Preliminary reports of these experiments have appeared (Xiao and Zečević, 1988; Zečević and Antić, 1992; Antić and Zečević, 1993a,b).

\section{Materials and Methods}

Preparation and dye injection. Experiments were carried out on identified neurons from the subesophageal and cerebral ganglia of the terrestrial snail, Helix aspersa. The ganglia were dissected from the animal and placed in a Sylgard-coated chamber containing Helix saline (mM): $80 \mathrm{NaCl}, 4 \mathrm{KCl}, 5 \mathrm{MgCl}_{2}, 5 \mathrm{CaCl}_{2}, 5$ Tris- $\mathrm{HCl}$ adjusted to $\mathrm{pH}=7.8$. The thick connective tissue was mechanically removed and the ganglion treated with a $0.5 \%$ trypsin solution (Sigma, Type IX) for 5-10 min at room temperature followed by $10 \mathrm{~min}$ exposure to a $0.5 \%$ solution of trypsin inhibitor (Sigma, Type II-S). After enzyme treatment, the thin connective tissue directly covering the neurons was carefully removed.

Neurons were impaled under visual control. The tip of the microelectrode was backfilled with near-saturated solution of a voltage-sensitive dye dissolved in distilled water. For example, we used a $3 \mathrm{mg} /$ $\mathrm{ml}$ dye solution of the styryl JPW 1114 that saturates at about $5 \mathrm{mg} / \mathrm{ml}$. The dye solution was filtered before filling the electrodes to eliminate microscopic particles that tend to block the tip of the electrode during pressure injection. We used Millex- $\mathrm{GV}_{4}$ filters with $0.22 \mu \mathrm{m}$ pore size (Millipore, Bedford, MA).

Dyes were injected by applying repetitive, short pressure pulses to the microelectrode using a Picospritzer (General Valve Corp., Fairfield, $\mathrm{NJ})$ The microelectrode tip size, pressure settings, and pulse duration were adjusted for each dye (pressure was varied between 5 and $60 \mathrm{psi}$, pulse duration between 1 and $50 \mathrm{msec}$, and microelectrodes ranged from 2 to $10 \mathrm{M} \Omega$ when filled with $3 \mathrm{M} \mathrm{KCl}$ ).

After the injection was completed, several preparations were incubated at different temperatures $\left(5,15,20,25\right.$, and $\left.30^{\circ} \mathrm{C}\right)$ for different periods (1-72) hr to determine the optimal conditions for the spread of a particular dye into the distal processes. For example, we found that the spread of the fluorescent styryl dye JPW 1114 , over a $1 \mathrm{~mm}$ long axonal branch, was not complete after $5 \mathrm{hr}$ at $15^{\circ} \mathrm{C}$. An improvement in the signal-to-noise ratio was obtained after $12 \mathrm{hr}$, but extending the incubation period further did not produce an additional improvement. We did not observe differences in the spread of the dye at 15,20 and $25^{\circ} \mathrm{C}$. The spread was noticeably slower at $5^{\circ} \mathrm{C}$. Incubation at $30^{\circ} \mathrm{C}$ for 2-3 hr did not noticeably improve the spread of this dye and longer incubations at $30^{\circ} \mathrm{C}$ were damaging to the preparation. For this dye, we routinely incubated the preparation at $15^{\circ} \mathrm{C}$ for $12 \mathrm{hr}$ before making optical recordings.

Absorption experiments were performed on identified neurons from subesophageal ganglia from young $(0.5 \mathrm{gm})$ animals to insure high transmitted light intensity. Ganglia from older animals were less transparent. The sensitivity of fluorescence measurements, using epiillumination, depended on finding a cell of suitable anatomy. All fluorescence measurements were performed on the identified giant metacerebral interneuron (Kandel and Tauc, 1965). The cell body of this neuron is situated on the ventral surface of cerebral ganglion and its main axonal branches project into the commissure and peripheral nerves, also running on the surface. There are two symmetrical cells in every preparation. Figure 1 shows a metacerebral interneuron injected with JPW 1114, as observed under epifluorescence. The ganglion was pinned in such a way that the long axonal branches are in one plane of focus for the $10 \times / 0.4$ NA objective that was used for optical recording.

Optical recording. We used the recording system for fast, multisite optical monitoring of membrane potential changes. Detailed descriptions of the method have appeared in Cohen and Lesher (1986) and Wu and Cohen (1993). A schematic diagram of the apparatus is shown in Figure 2. The preparation was positioned on the stage of a microscope and the image of the stained cell projected onto the photodiode array positioned at the primary image plane. A tungsten-halogen bulb was a light source in all experiments. Interference filters or filter-combinations were used to select the appropriate wavelength of light in absorption and fluorescence measurements. Transmitted light was used for absorption measurements and epiillumination for fluorescence. The optical signals were recorded by 124 elements of a $12 \times 12$ photodiode array (Centronix Inc., Newbury Park, CA). The output current of each diode was converted to voltage and individually amplified. High frequency noise in the recording was limited by the $1 \mathrm{kHz}$ cutoff frequency of the low-pass filter (four-pole Bessel filter). An RC filter with a cutoff frequency of $1.5 \mathrm{~Hz}$ was used to limit low-frequency noise. The recording apparatus (Wu and Cohen, 1993) was modified to limit the illumination time to a necessary minimum. An electronic switch was included in the 


\section{ABSORPTION}

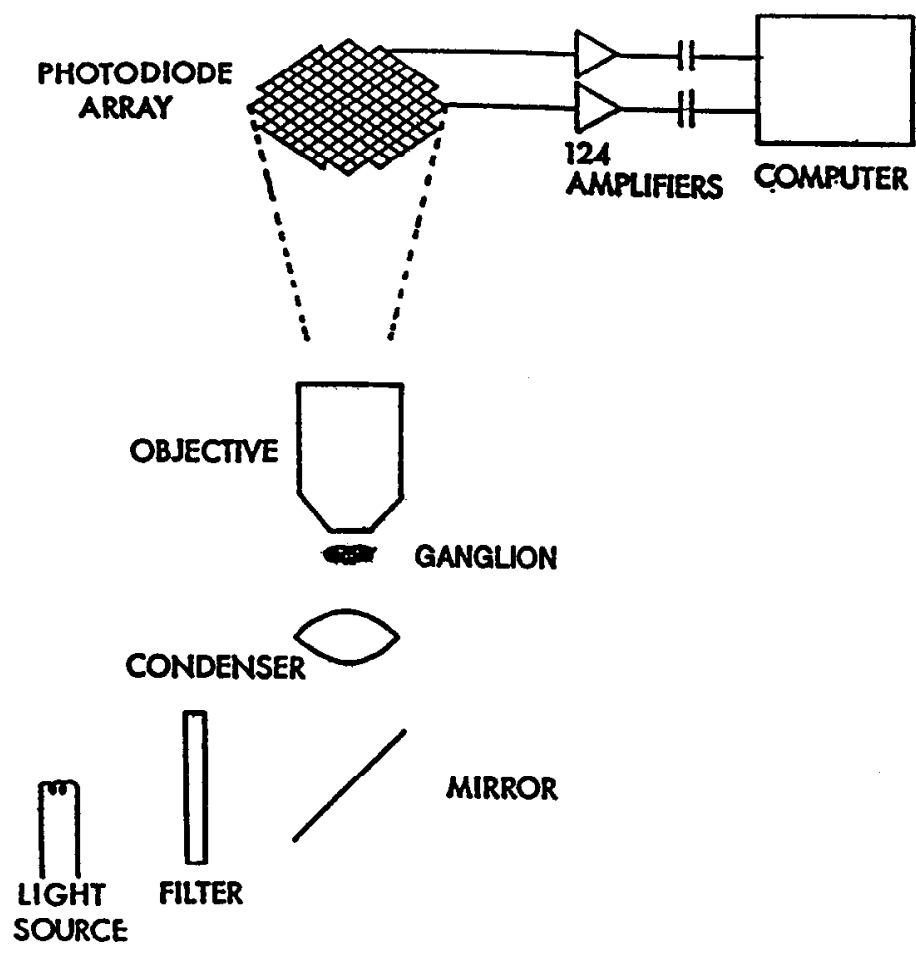

\section{FLUORESCENCE}

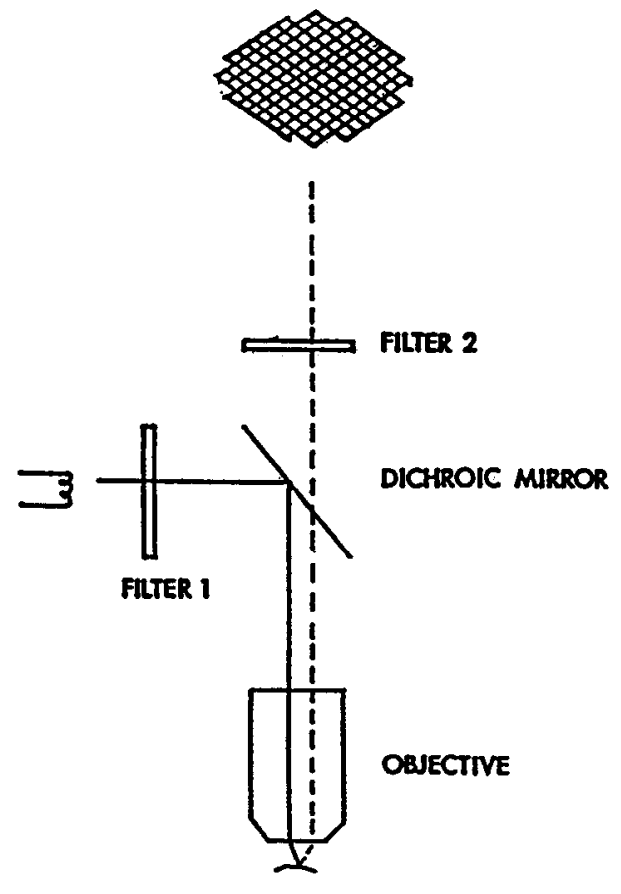

Figure 2. Schematic drawing of the optical apparatus for absorption and fluorescence measurements. The photodiode array was placed at the plane where the objective makes a real, magnified, and inverted image. The illumination intensity was increased by about $50 \%$ by overrunning the $12 \mathrm{~V}, 100 \mathrm{~W}$ tungsten-filament lamp ( $9 \mathrm{~A}$ instead of $8.3 \mathrm{~A}$ ). In absorption measurements, light from a tungsten-filament lamp was heat filtered, passed through an appropriate interference filter, and focused on the preparation using a modification of Kohler illumination and ordinary light microscopy. In fluorescence measurements, epillumination with a $10 \times, 0.4 \mathrm{NA}$, long working distance, objective was used. The preparation was illuminated with the green portion (interference filter $520 \pm 45 \mathrm{~nm}$ ) of the output of a tungsten-filament lamp. Fluorescence emission from the preparation was selected by means of a dichroic mirror and an appropriate barrier filter (Schott RG610). The output of each detector was individually amplified, filtered (four-pole Bessel filter, Wu and Cohen, 1993), multiplexed, digitized, and stored in a Motorola VME bus computer also used to analyze and display the data.

amplifier circuit that allowed an AC coupling time constant of about 1 msec to be enabled under computer control every time the shutter controlling the light was opened. Amplifiers would settle to baseline in about $10 \mathrm{msec}$. The time constant was then switched back to that desired for recording, and the recording started. In this way, we were able to close the shutter between trials and keep the light off except for the $150 \mathrm{msec}$ recording interval for each trial. Amplifier outputs were digitized at a $1 \mathrm{kHz}$ sampling rate.

In a typical experiment, the cell was stimulated by passing current through a microelectrode to produce action potentials or electrotonic, subthreshold responses, while simultaneously recording optical signals from multiple sites on the neuron. When signal averaging of optical traces was required, the data acquisition program determined the time of the peak of the electrically recorded action potential, and this data point was used as the reference point for averaging. This was necessary because the delay between the onset of the stimulus delivered through the microelectrode in the soma and the evoked action potential was not perfectly constant.

\section{Results}

The first series of experiments ware carried out using absorption measurements because absorption dyes were substantially better in terms of photodynamic damage as compared to fluorescence dyes. We tested analogues of the most successful absorption dyes known from extracellular application (Ross et al., 1977; Cohen and Lesher, 1986; London et al., 1987; Zecevic et al., 1989). A total of 30 dyes were tested (20 pyrazolone-oxonol molecules, 5 merocyanine dyes, 3 barbituric-acid oxonol dyes, and 2 styryl dyes; see Fig. 3 legend) after intracellular application. The best results were obtained with two positively charged pyrazolone-oxonol dyes (designated JPW1177 and JPW1245), and two negatively charged merocyanine dyes (designated WW375 and JPW1124). However, the signal-to-noise ratio for the best dyes in these series, in recording action potential related signals from the cell soma, varied from $1-4$ in single trials. The signal-to-noise ratio was determined as the size of the action potential $(70-80 \mathrm{mV})$ related optical signal relative to the peakto-peak noise in the optical trace. Thus, absorption signals were not large enough to allow monitoring voltage transients from neuronal processes without extensive averaging. The low sensitivity of absorption measurements will be discussed below.

Fluorescence measurements are more effective than absorption measurements in the situation where the image of the object (e.g., thin process) is much smaller than the size of a photodetector (Grinvald et al., 1982; Cohen and Lesher, 1986). When the modified amplifiers (Wu and Cohen, 1993) were used to limit the illumination period to the necessary minimum and improve the dark noise we began fluorescence measurements. We found that JPW1114 was better than the three other fluorescent styryl molecules we tested (RH461, RH437, and JPW1063) as deter- 


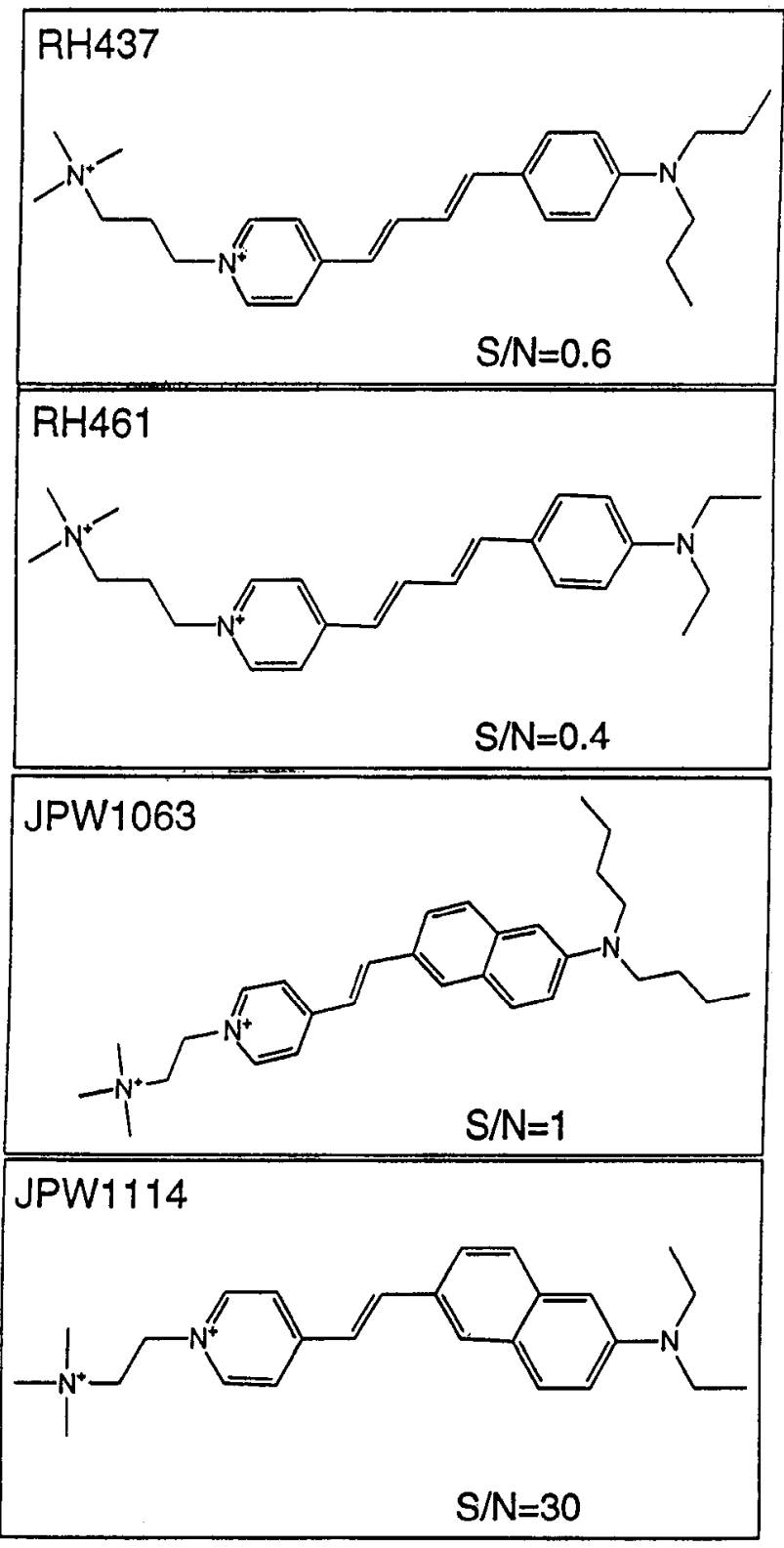

Figure 3. The structures of the doubly positively charged styryl dyes synthesized specifically for intracellular application by R. Hildesheim and A. Grinvald (RH461 and RH437) and by J. Wuskell and L. Loew (JPW1063 and JPW1114). JPW1114 is an analog of the widely used voltage-sensitive dye di-4-ANEPPS (Loew et al., 1992). These four molecules were tested in fluorescence measurements and the single trial signal-to-noise ratio $(\mathrm{S} / \mathrm{N})$ is indicated for each dye. Additional dyes, tested in the absorption mode, are listed below with the single tria signal-to-noise ratio in recording action potential signals from cell soma given in parentheses. Structural formulas are available from the authors Pyrazolone-oxonol dyes: 1. RGA509 (0.6); 2. RGA565 $(<0.2) ; 3$. RGA574 (<0.2); 4. RGA577 (<0.2); 5. RH155 (<0.2); 6. RH479 $(<0.2)$; 7. RII482 $(<0.2) ; 8$. JPW1037 $(<0.2) ; 9$. JPW1038 $(<0.2) ; 10$. JPW $1039(<0.2) ; 11$. JPW $1044(<0.2) ; 12$. JPW $1177(4) ; 13$. JPW1241 (1); 14. JPW1245 (3); 15. JPW1247 (<0.2); 16. JPW1249 (<0.2); 17. JPW1269 $(<0.2) ; 18$. JPW1278 $(<0.2) ; 19$. JPW1286 $(<0.2) ; 20$. JPW2004 (<0.2). Merocyanine-rhodamine dyes: 21. NK2367 (4); 22. JPW1124 (4); 23. JPW1299 (<0.2) 24. WW375 (3); 25. WW765 $(<0.2)$. Barbituric acid oxonol dyes: 26. JPW1281 $(<0.2) 27$. WW781 $(0.5)$; 28. WW808 $(<0.2)$. Styryl dyes: 29 . RH437 (0.2); 30. RH461 $(<0.2)$. Dyes designated RGA and WW are synthesized by R. Gupta, C. Wang, and A. Wagooner, Center for Fluorescence, Carnegie Mellon University, Pittsburgh, PA. Dyes designated RH are synthesized by $\mathbf{R}$. Hildesheim and A. Grinvald, Weizmann Institute, Rehovo, Israel. NK dyes are available from Nippon Kanko-Shikiso Kenkyusho Co. Ltd. mined by signal-to-noise ratio. The structures of these dyes are shown in Figure 3, together with the single trial signal-to-noise ratio we obtained. JPW1114 was used to obtain results shown in all of the subsequent figures.

Figure $4 A$ illustrates the shape of the giant metacerebral neuron in the intact ganglion and how its image was projected onto the array of photodetectors. Since the drawing was done from the unfixed preparation, there was no shrinkage artifact; the detectors in the array are matched correctly with the cell image. The geometry of the neuron is such that both cell soma and long axonal branches are located in the same plane and are in focus for the $10 \times$ objective. The image of the neuron was projected onto the $12 \times 12$ array of photodiodes such that each detector received light from a square area of $112 \times 112 \mu \mathrm{m}$ in the object plane. Changes in fluorescent light intensity were detected by each of 124 elements of the array during the time the neuron was stimulated to produce action potentials.

It is important to know how accurately the voltage-sensitive dye signals represent electrical events. For many voltage-sensitive dyes, absorption and fluorescence changes are both fast and linear with membrane potential changes in the squid giant axon (Gupta et al., 1981; Cohen and Lesher, 1986; Salzberg et al., 1993). We confirmed this conclusion on metacerebral giant neurons injected with voltage-sensitive dyes by comparing electrical signals with optically recorded action potentials. Figure $4 B-E$ shows optical recordings from selected detectors on an expanded time scale; the optical signals closely follow the waveform of electrically recorded action potentials.

The fractional change in fluorescence light intensity from neuronal processes was of the order of $2 \times 10^{-2} / 100 \mathrm{mV}$. While the signal size expressed as the fractional change in light intensity was roughly the same along the length of the axonal branches, the fractional change was always smaller in the soma regions. Presumably, the background fluorescence from dye not bound to surface membrane was higher in the soma. The soma might have higher proportion of internal membranes per unit volume in comparison to the axon, and/or a less favorable surface to volume ratio. Also, the signal-to-noise ratio from the soma was degraded by vibration noise in the recording. The light intensity from the brightly fluorescent soma was high enough so that noise due to mechanical vibrations in the system was higher than the electrical dark noise that was dominant in the recordings from processes.

With the sensitivity of the dye described and the level of noise in our recording system, relatively good signal-to-noise ratios could be obtained in single trial recordings from most of the processes. However, the most distal regions required averaging to improve signal-to-noise. Usually 4-25 trials were averaged for action potential signals and $20-100$ trials for $10 \mathrm{mV}$ subthreshold responses. Figure $4 B$ illustrates a good signal-to-noise ratio in single-trial recording from $6 \mu \mathrm{m}$ axonal branch some $300 \mu \mathrm{m}$ away from the soma. Figure $4 C$ shows the improvement in signal-to-noise when nine trials were averaged in recordings from the same location, and Figure $4 D$ shows the action potential signal from a more distal thin $(3 \mu \mathrm{m})$ axonal branch some $600 \mu \mathrm{m}$ away from the soma. Figure $4 E$ illustrates that it is possible, with signal averaging, to achieve a reasonable signal-

$\leftarrow$

JPW dyes are synthesized by J. Wuskel and L. Loew, University of Connecticut Health Center, Farmington, CT (Gupta et al., 1981; Cohen and Lesher, 1986; Loew et al., 1992). 


$$
\begin{aligned}
& \text { AXON (6,um) } \\
& \text { Distance: } 300, \mathrm{um}
\end{aligned}
$$<smiles>C=[OH+]</smiles>

ELECTRICAL TR=9

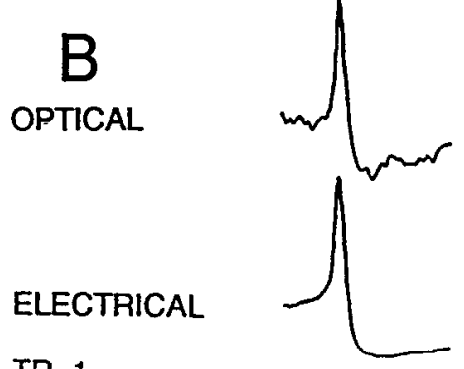

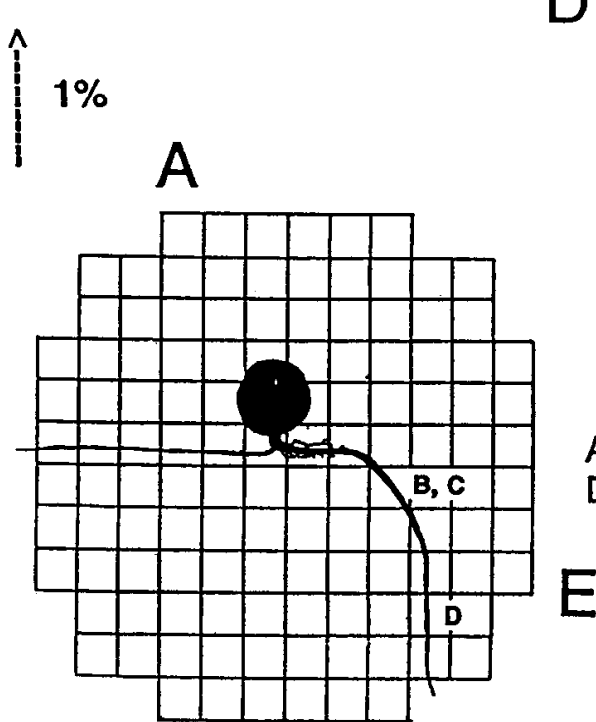

\author{
AXON $(3, u m)$ \\ Distance: 600 ,um
}

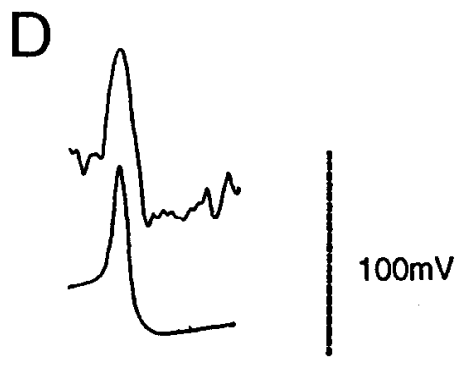

AXON (3 ,um)
Distance: $400, \mathrm{um}$

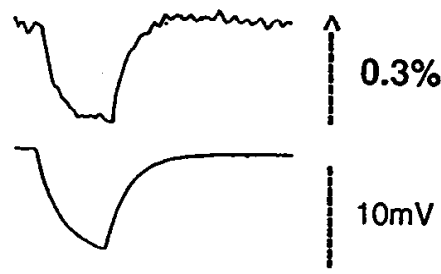

$\mathrm{TR}=100$

$100 \mathrm{~ms}$

Figure 4. A, Outline of the 124 element photodiode array superimposed over a drawing of the fluorescent image of a live giant metacerebral neuron as projected by a $10 \times, 0.4 \mathrm{NA}$ objective with a $1.25 \times$ auxiliary lens. Fach diode receives light from $112 \times 112 \mu \mathrm{m}$ area in the object plane. $B-E$, Comparison of electrical and optical recordings. The optical signals were from different locations on the neuron as labeled on the outline of the array $(B-D)$. Recordings shown in $E$ are from a different experiment. The electrode recordings were all from the soma; $100 \mathrm{mV}$ and $1 \%$ scale bar applies to $B-D$. Number of trials averaged $(T R)$ is the same for $C$ and $D$. Axon diameters and distances of recording locations from the soma are given in $\mu \mathrm{m}$.

to-noise ratio in recording relatively small, electrotonic responses from distal thin processes.

Although the optical signals are similar to the microelectrode measurements, there are some discrepancies due to filtering the optical signals. Figure 5 shows, on an expanded time scale, a comparison of the unfiltered microelectrode recordings from the soma with the optical signals filtered to a different degree to eliminate high-frequency noise. Both action potentials and subthreshold hyperpolarizing response are shown. The optical signals were recorded from the proximal axonal regions adjacent to the cell body to eliminate propagation delays. The shape of the optical signal (Fig. $5 A-C$ ) was unaffected by low-pass filtering with cutoff frequency set to 1 and $0.5 \mathrm{kHz}$, and very little Inodified at $0.3 \mathrm{kHz}$. The delay in the optical signal introduced by filtering is the function of the cutoff frequency and was most pronounced at $0.3 \mathrm{kHz}$. This delay is a constant that does not depend on signal characteristics and can be ignored, since our conclusions are based on comparing optical signals with the same filtering. This is illustrated in Figure 5, $E$ and $F$, where filtered optical recordings from two adjacent regions appear identical at both 0.5 and $0.3 \mathrm{kHz}$ corner frequencies. However, all our subsequent records were filtered with a high-frequency cutoff of $1 \mathrm{kHz}$ to minimize the delay introduced by the filter. This conservative approach is based on the fact that the delay may not be exactly the same for each individual channel in the multichannel recording system that we use. We have not deter- mined the extent of the variation in filter-induced delays in different channels, but the results shown in Figure 5, $E$ and $F$, indicate that this variation is small. If this can be demonstrated for all channels, it would be more appropriate to use corner frequencies of 0.5 or $0.3 \mathrm{kHz}$ to optimize the signal-to-noise ratio. In addition to analog filtering, digital smoothing was used (1-2-1 smoothing routine, London et al., 1987) but only to an extent that did not change timing information in the signal. After the spikes in Figure 5, the optical signal appears to decrease more rapidly than the electrical signal. This was an effect of the high-pass filter (in the optical amplifiers) used to limit low-frequency noise. These differences between electrical and optical signals could be largely removed by digitally applying a similar high-pass filter to the electrical signals (not shown).

The extent of pharmacological effects and photodynamic damage induced by JPW1114 was explored. Previous work has shown that certain voltage-sensitive dyes have little or no effect on the physiological parameters of the action potential when applied from the outside within a limited concentration range (Ross et al., 1977; Gupta et al., 1981). We found that action potentials evoked in the soma were not affected by intracellular dye injection in amounts sufficient to stain distal processes. Howcver, prolonged injections sometimes resulted in sudden disintegration of the cell. We believe that this was the result of mechanical damage induced by applying pressure through the microelectrode. 

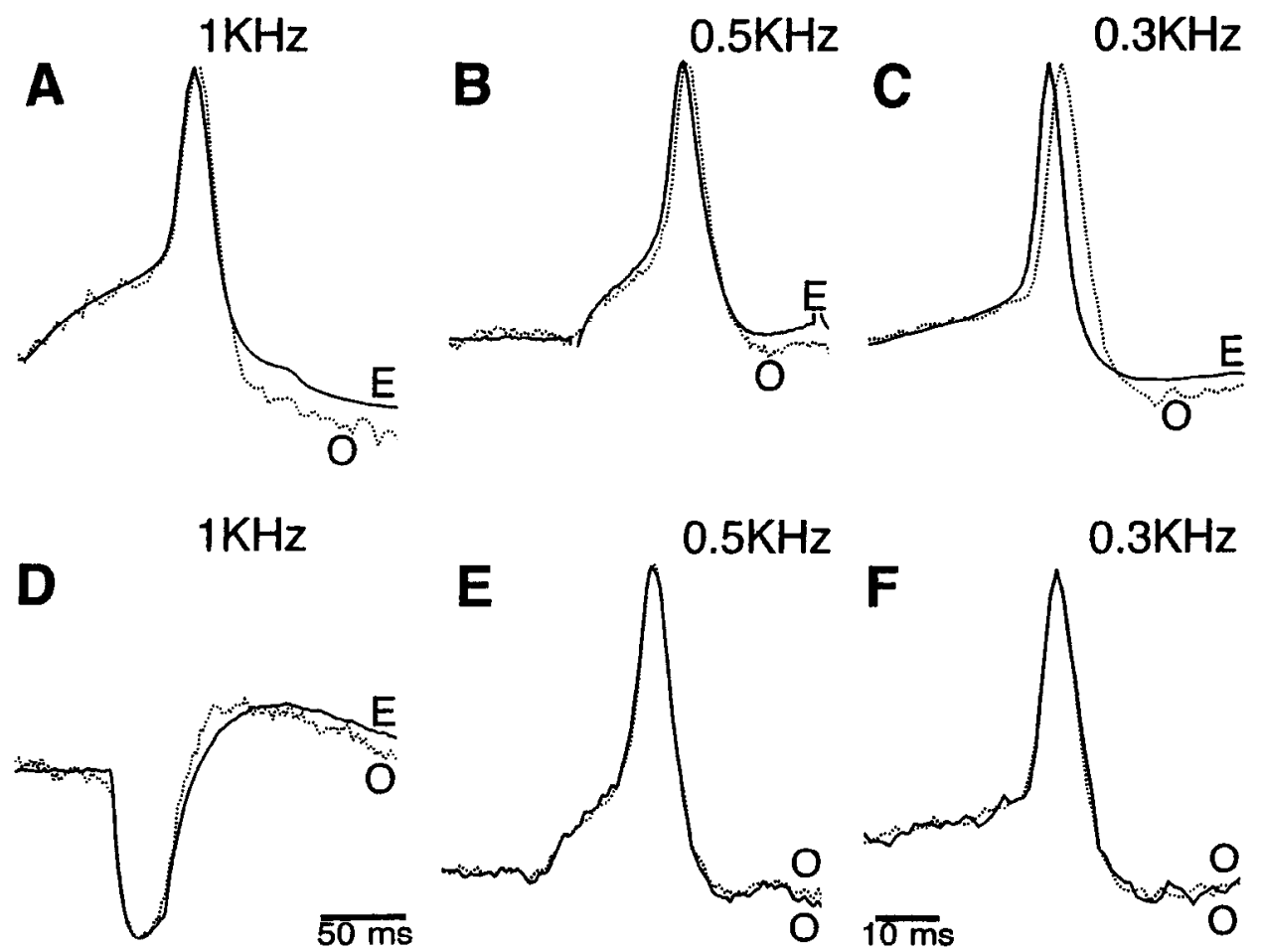

Figure 5. The effect of the low-pass filter on the shape and size of optical signals. Traces from microelectrode recordings and optical detectors were scaled to the same height by adjusting the baseline level and $y$-axis gain for each trace separately. Small variations in baseline levels in this scaling procedure had little effect on calculated delay values. The same procedure was used in Figures 7 and 8 . Filtering optical signals with a low-pass cutoff frequency of $1,0.5$, and $0.3 \mathrm{kHz}$ reduced the noise while the distortion of the action potential shape was negligible $(A-C)$. Lowpass Bessel filters exhibit a delay between the input signal and the output signal that is inversely proportional to the cutoff frequency and is most obvious at $0.3 \mathrm{kHz}(C)$. The shape of the hyperpolarizing signal was also unaffected by low-pass filtering at a $1 \mathrm{kHz}$ frequency $(D)$ ). $E$ and $F$ show superimposed optical recordings from two adjacent regions that appear identical if filtered at the same corner frequency $(0.5 \mathrm{or} 0.3 \mathrm{kHz})$, indicating that the delay characteristics of the individual channel filters are similar. The faster time scale applies to the action potential recordings and the slower time scale to the hyperpolarizing response. $E$, electrical recording; $O$, optical recording.

Experiments on marine mollusks Navanax and Aplysia (Londun et al., 1987; Zečević et al., 1989; Parsons et al., 1991) showed that while some oxonol absorption dyes caused little or no photodynamic damage even after prolonged illumination, bleaching and photodynamic damage were substantially more rapid with fluorescent dyes. Also, in our initial experiments on Helix neurons using fluorescent styryl dyes, we were limited in averaging optical signals to approximately 20 trials by photodynamic damage that caused a pronounced reduction in the membrane input resistance and progressive decline in action potential amplitude. In those experiments neurons were exposed to high intensity light from the start of the averaging series to the end of the last trial. With amplifiers modified to allow intermittent illumination and limit the illumination time to a necessary minimum (see Materials and Methods), we saw very little photodynamic damage. It was possible to average 100 trials with no noticeable change in action potential amplitude or waveform. The introduction of dark intervals in the series of measurements might have reduced photodynamic damage not only by reducing the absolute time the cell was exposed to high intensity light but also by introducing intermittent "recovery" periods (Bonhoeffer and Staiger, 1988).

\section{Multiple site recording}

Figure 6 shows a sinultaneous multisite optical recording. The fluorescent light intensity was recorded by each of the 124 elements of the array during the time the neuron was stimulated to produce action potentials. Each trace in the figure represents the output of one photodiode. The full array is not represented; the two top rows of detectors do not have any signals and are not included in the figure. The same recording is represented on the figure with three different $y$-axis scales to illustrate both the bigger signals from large processes and somatic regions and smaller signals from thin axons. Optical signals corresponding to evoked action potentials were clearly recorded from both soma and processes. In Figure $6, B$ and $C$, most of the detectors appear flat; this is the result of deleting the data to flatten both traces with no signals and traces with signals that would be too large, in order to emphasize the smaller signals. In Figure $6 A$, traces were not conditioned except one from a detector over the soma. The best signal-to-noise ratio was obtained from the detectors that appear to be over regions of relatively thick proximal axonal branches. The signal-to-noise ratio declined with increasing distance from the soma and with diameter of axonal branches. As evident from the figure, the optical signals were found in the regions of the array that correspond closely to the geometry of the cell. Since this was the only cell stained in the ganglion, the source of the signal is known and we can safely attribute optical signals to potential transients in specific regions of the soma and processes of the stimulated cell.

While the evidence presented above (Fig. 5) shows that the dye signal is proportional to the membrane potential change, it is not possible to determine the actual voltage change from these optical recordings. The optical signal size depends not only on the amplitude of membrane potential change but also on the 
Figure 6. Optical recording from elements of the photodiode array when the giant metacerebral cell was stimulated to produce action potentials (16 trials averaged). Each trace represents the output of one diode for $70 \mathrm{msec}$. The traces are arranged according to the disposition of the detectors in the array. The same data are represented with increasing y-axis scale in $A-C$. In $B$ and $C$, part of the data were deleted to improve clarity. See explanation in the Results. Optical signals correspond closely to the geometry of the cell as outlined in the figure.

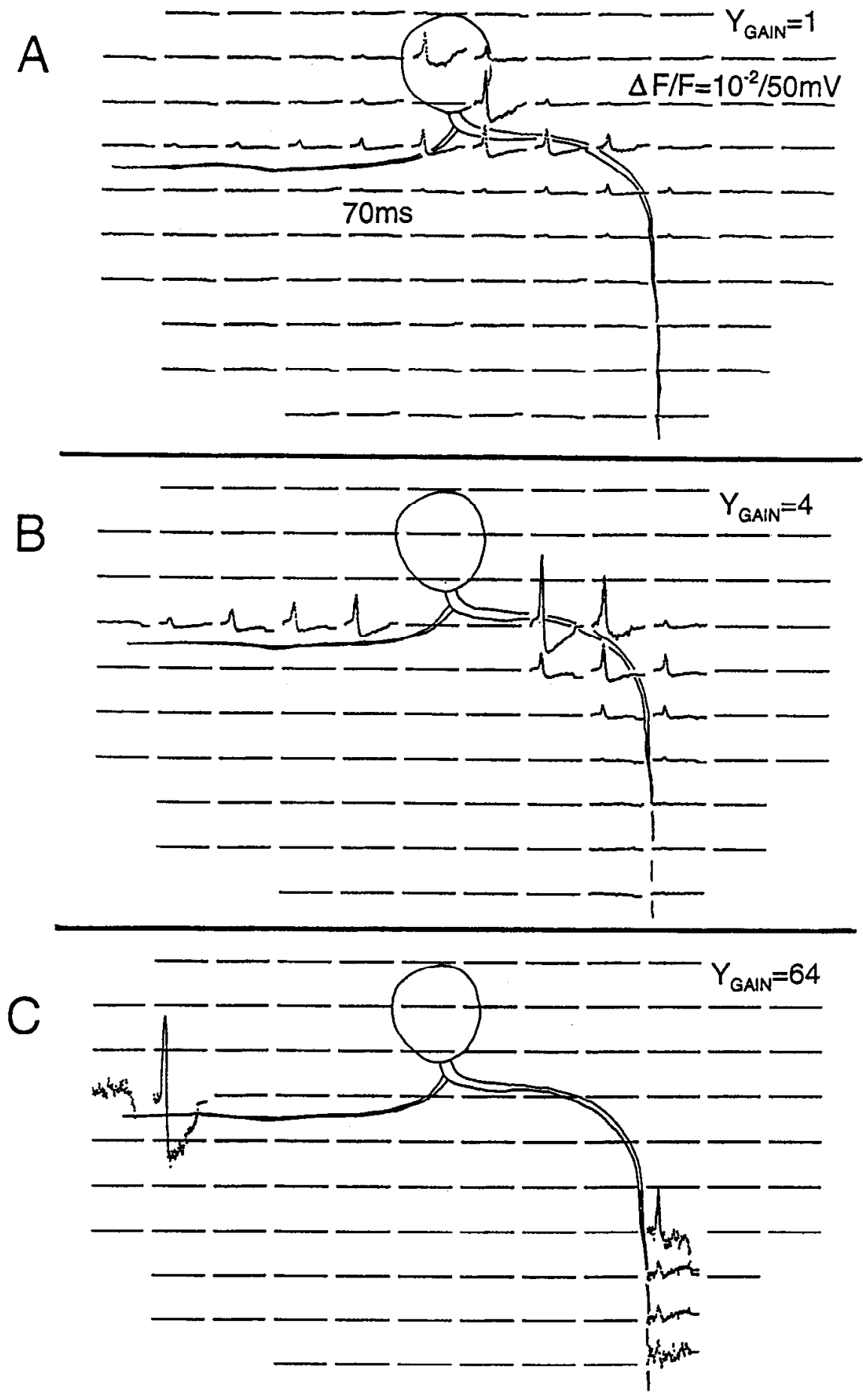

amount of dye bound to the active membrane and on the active membrane area imaged onto each detector. Both the amount of dye bound to the active membrane and the active membrane area are difficult to determine and should vary from one region of the neuron to the next. We presume that the variation in these parameters are responsible for the marked differences in optical signals corresponding to action potentials from different regions of the cell. However, if we assume that the action potential has the same amplitude throughout the soma and axonal branches, we can then scale the optical signals to the same height, and use this scaling factor to determine the size (in $\mathrm{mV}$ ) of subthreshold signals (see below).

\section{Action potential propagation}

In the experiment illustrated in Figure 7 , the image of a long axon was projected onto a single row of 12 detectors. The recordings from selected photodiodes (indicated in the figure) report action potentials from different positions along the axon. Traces from different detectors, scaled to the same height, are superimposed together with the recording from the most proximal axon region. The increasing delay of each subsequent record is due to the propagation delay. The proximal region of the axon, between the first and the fourth pixel, appears to be the site of action potential initiation. Because no delay has been recorded between these two pixels, which are $224 \mu \mathrm{m}$ apart, the simplest 


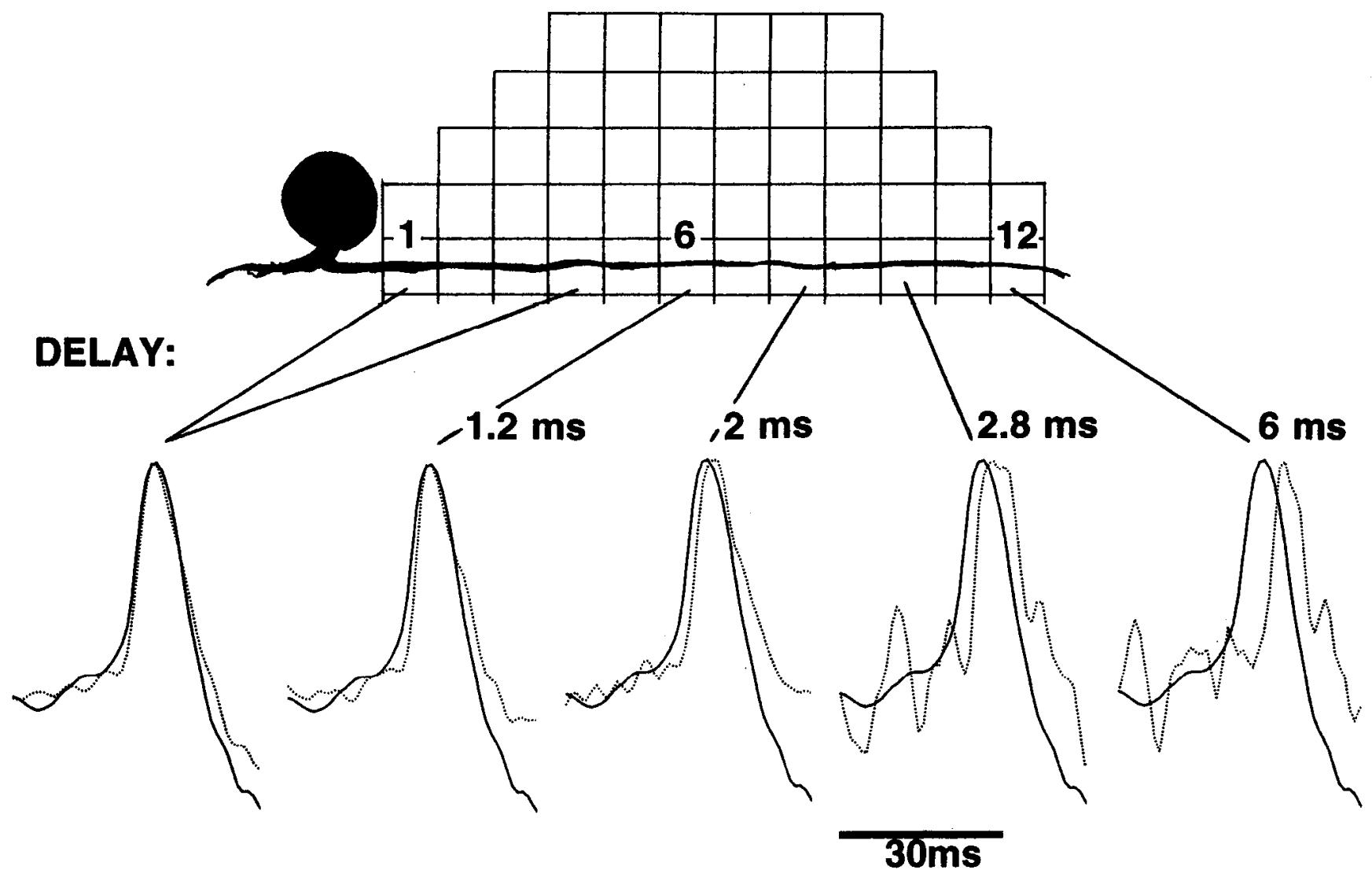

Figure 7. Optical recordings of action potential signals from different locations along the axon of the giant metacerebral cell. Twenty-five trials averaged. Traces from different detectors, scaled to the same height, are superimposed together with the same recording from the most proximal axon region. The increasing delay between the signal from the most proximal axonal segment and records from more distal parts reflects the propagation velocity. Different axonal segments appear to have different propagation velocity.

explanation is that the action potential evoked by soma stimulation originates in this region.

If we calculate the propagation velocity across the array (from pixel 4 to 12), the overall velocity was $0.15 \mathrm{~m} / \mathrm{sec}$. However, different axonal segments appear to have different propagation velocities. The highest velocity $(0.28 \mathrm{~m} / \mathrm{sec})$ was detected in the axonal region between pixel 6 and 10, and the slowest $(0.07$ $\mathrm{m} / \mathrm{sec}$ ) in the most distal region, between pixels 10 and 12 . In principle, the accuracy of these measurements based on the comparison of time-to-half-amplitude, is better than $1 \mathrm{msec}$ sampling interval because the measurements are made along a rapidly changing signal (Ross and Krauthamer, 1984). However, the signal-to-noise ratio in recordings from distal parts of the long axonal branch in this experiment (locations 10 and 12, Fig. 7) was not good enough to exclude the possibility of errors in calculating delays. In spite of possible errors, the experiment shown in Figure 7 is important since this was the longest axonal process (1344 $\mu \mathrm{m})$ that we were able to analyze. The result indicates that more distal, thin axonal regions might be characterized by substantially lower propagation velocities than the proximal parts.

In the similar measurements from two other metacerebral interneurons, the signal-to-noise ratio was improved by averaging 64 trials (Fig. 8A,B). In the experiment shown in Figure $8 A$, the first action potential occurred in an axonal segment about 500 $\mu$ In away from the soma, in the region between pixel 2 and 5 . The spike propagated back toward the soma with the velocity of $0.53 \mathrm{~m} / \mathrm{sec}$ between locations 5 and 8 , and was recorded with a delay from the other axonal branch (location 12). The propa- gation velocity calculated between pixels 9 and 12 was 0.27 $\mathrm{m} / \mathrm{scc}$. Since the signal-to-noise ratio is relatively good in this experiment, the nonuniform propagation velocity appears to be real. As a result of different excitability of the separate axonal regions, the action potential initiated by depolarizing the soma appeared first in the remote part of one axonal branch and only after a clear delay in the other axonal branch adjacent to the soma (location 12 in Fig. 8). However, the origin of the spike recorded from the thicker axonal branch (location 12) in Figure $8 A$ is not clear. It could have originated from the propagation of the action potential evoked in a distal part of the thinner axonal branch. Alternatively, it might be the result of activating a separate action potential in the thicker axonal branch. In fact, the results of the measurements from another neuron, shown in Figure $8 B$, indicate that two separate spikes are evoked in the two axonal branches by soma stimulation. Recordings from individual detectors from locations 1 and 2 show propagation of the action potential from a distal spike trigger zone toward the cell body with the velocity of $0.25 \mathrm{~m} / \mathrm{sec}$ in the thinner axonal branch. Recordings from locations 3 and 4 show that the action potential in the thicker axonal branch also propagates toward the soma from a more distal site of origin, with a velocity of only $0.16 \mathrm{~m} / \mathrm{sec}$. This result was also found in three other cells. It is clear that there must be at least two separate spike trigger zones in the metacerebral interneuron and that the propagation velocity is different in different axonal segments. The simplest explanation for the decrease in propagation velocity would be that the decrease in axon diameter was responsible (as predicted by clas- 
A

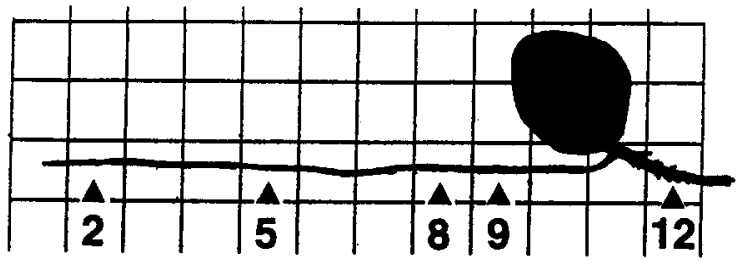

DELAY:

Oms

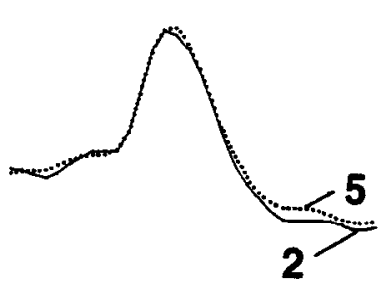

B
$0.6 \mathrm{~ms}$

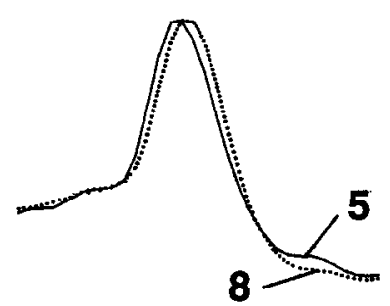

8
$1.3 \mathrm{~ms}$

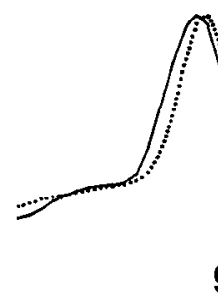

$2.3 \mathrm{~ms}$

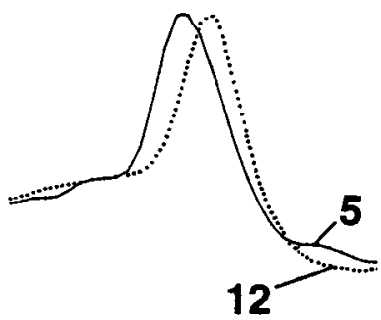

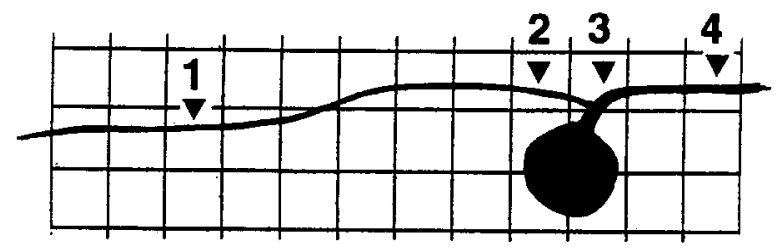

\section{DELAY:}
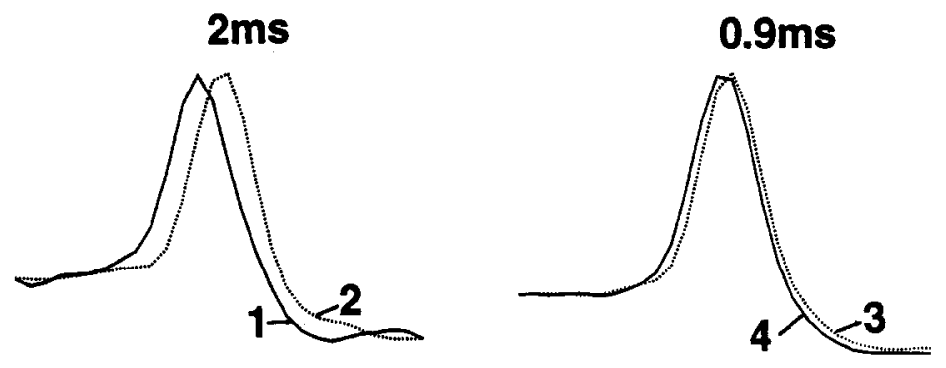

$15 \mathrm{~ms}$

Figure 8. Optical recording of action potential signals from different locations along the axonal branches of two metacerebral interneurons. Signalto-noise ratio improved by averaging 64 trials. $A$, Different axonal segments appear to have different excitability and different propagation velocity. $B$, The direction of action potential propagation and propagation velocity determined by comparing recordings from different locations on two main axonal branches. In both axons, the spike propagated toward the soma from the more distal site of origin indicating the existence of two separate spike trigger zones.

sical cable equations, Hodgkin and Rushton, 1946). However, cable theory predicts that propagation velocity is proportional to the square root of the diameter for nonmyelinated axons if all other factors remain the same. Thus, cable theory would require that axon diameter be reduced by a factor of 16 to account for the differences in propagation velocity we detected in the experiment shown in Figure 7. Measurements of axon diameter show that the most distal axonal branches were thinner than the proximal regions by a factor of 4 at the most. In the experiment shown on Figure $8 B$, the velocity was lower in the thicker axonal branch. Thus, it appears that other factors determining the space constant and propagation velocity must be involved.

\section{Spread of a hyperpolarizing stimulus}

Multisite recording can also be used to follow the passive spread of local responses in the processes. An example of such an experiment, using the same cell shown in Figure 6, is illustrated in Figure 9. The cell was polarized by $10 \mathrm{mV}$ with a $30 \mathrm{msec}$ transmembrane current step to mimic a large inhibitory synaptic potential. This local response was recorded electrically by the microelectrode in the soma and optically by the array of detectors. The same data are shown with two different y-axis scales. The decline in amplitude of the optical signal with distance from the soma may result from several factors. First, it may be due to the fact that the axon is becoming thinner and less dye is 


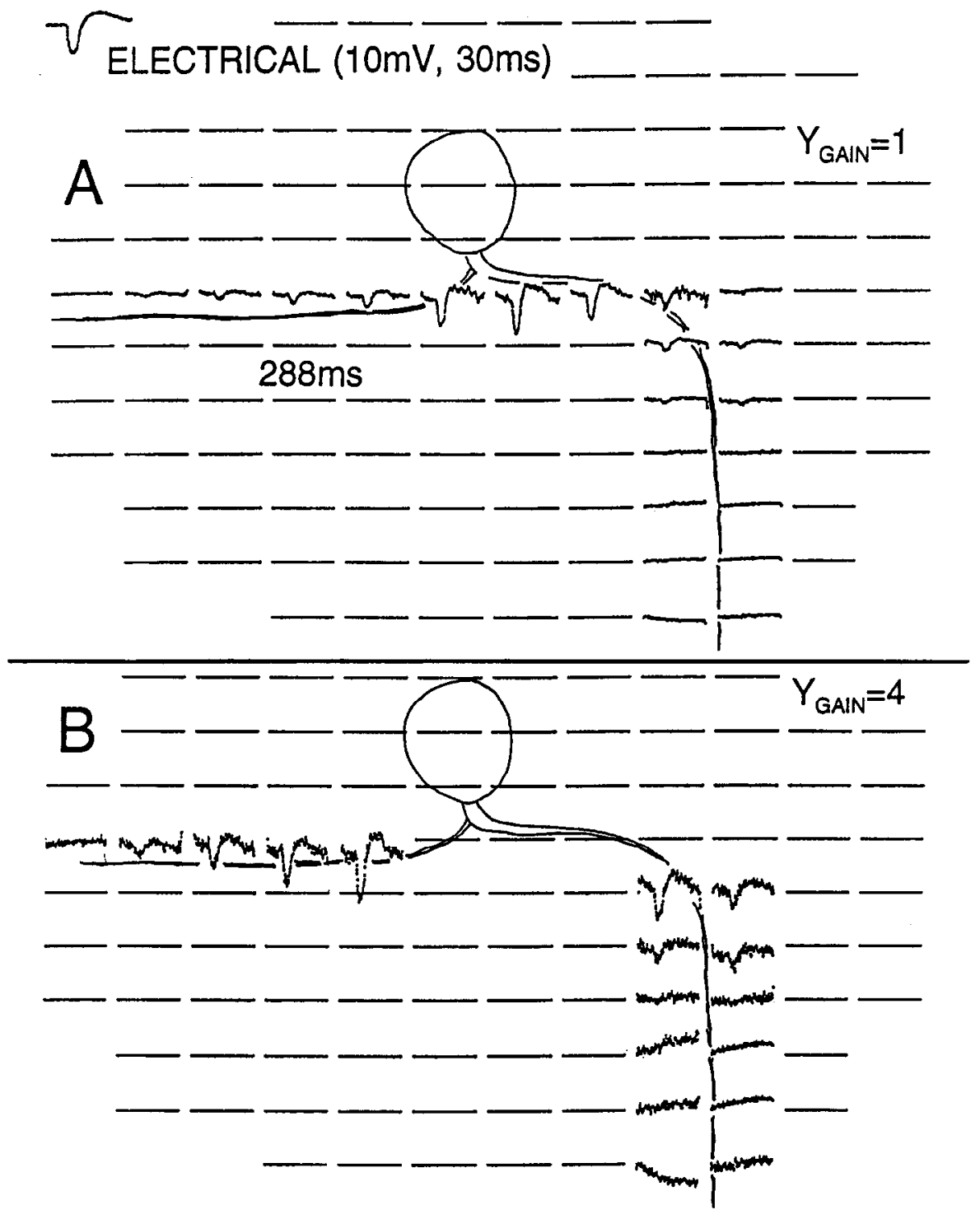

Figure 9. Optical recording during a hyperpolarizing voltage step induced by passing current in the soma. Twenty-three trials were averaged. This hyperpolarizing step is recorded electrically by the microelectrode in the soma (upper left corner in A) and optically. The same data are shown with two $y$-axis scales in $A$ and $B$. As in Figure 6 , some traces are flattened by the display program to improve clarity.

available to generate the signal. Second, the decline might be also due to the reduction of dye concentration per unit membrane area with distance from the place of injection (soma). And third, part of the decline is due to a reduction of the electrotonic potential change with distance. If we assume that the action potential in this cell has a constant amplitude along the axon, then the data of the type shown in Figure 6 can be used to determine the scaling factor for each detector.

In Figure 10, the optical recordings are scaled to reflect the relative amplitudes of the evoked hyperpolarization at different positions. The same signal from the most proximal region of the axon is superimposed with recordings from morc distal parts. In this experiment, we are dealing with potential transients that are about 10 times smaller in amplitude than the action potential and the signal-to-noise ratio is relatively low. Due to the relatively large noise in the recording from distal axonal regions, it is not possible to extract timing information from these data, nor is it possible to determine reliably the rate of decline with distance. However, the clear decline in the size of the scaled electrotonic hyperpolarizing response with distance was observed in the experiment shown in Figure 10 and in two other similar experiments. This result also implies that relatively modest im- provements in sensitivity will allow the direct analyses of the spread and summation of synaptic potentials in neuronal processes without the need for extensive averaging.

\section{Discussion}

These experiments were carried out to monitor directly the generation and spread of action potentials and subthreshold signals in neuronal processes. Optical methods with improved sensitivity for recording potential changes simultaneously at many places on neuronal arborization were used.

\section{Optical signals, pharmacological effects, and photodynamic damage}

Individual neurons were selectively stained by intracellular dye application. The first series of experiments was done using absorption measurements because, at the beginning, we were not technically prepared for efficient fluorescence measurements, and photodynamic damage was a serious problem. The available absorption dyes are not as photuloxic as fluorescent molecules (Cohen and Lesher, 1986). We think that our effort to use absorption probes was not successful because of the following technical difficulties in using absorption in recording from small 


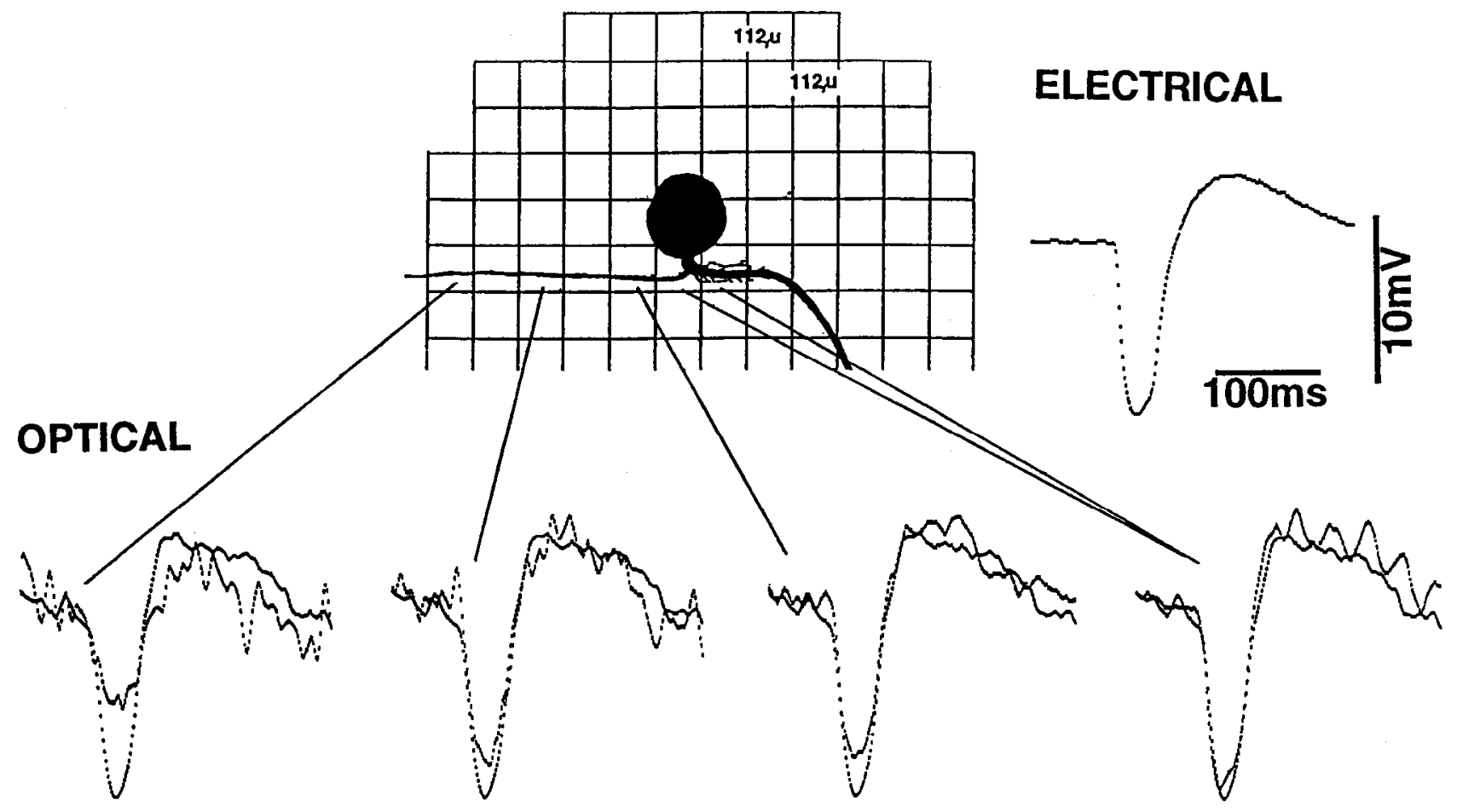

Figure 10. Optical signals, scaled to reflect relative amplitudes of the hyperpolarizing membrane potential change at different locations. Twentythree trials averaged. The signal from the most proximal region of the axon is superimposed with recordings from more distal parts. The size of hyperpolarizing response declines with distance.

processes. One difficulty arises because the fractional change in absorption measurements is directly proportional to the amount of the dye bound to membrane. If 10 times less dye is bound to a small process, the fractional change in transmitted light intensity is 10 times less. This makes it important to stain the membrane heavily. But there are limitations imposed by pharmacological effects when using too much dye. This limitation is especially severe in intracellular dye application; while it is relatively easy to adjust the dye concentration in extracellular application, it is not always possible to increase the amount of dye in distal processes by putting more dye in the soma. It is our experience that during acute dye injection a point is reached when the cell is damaged by additional dye. The amount of dye could be increased by using several injections separated by rest periods, but we did not test this possibility. The second difficulty with absorption measurements from small processes is that the signal-to-noise ratio is dependent on the ratio of the image size to the size of the detector. If the object image is smaller than the detector, the signal-to-noise is degraded. In trying to record simultaneously from different places on the axonal branches and dendritic tree, an objective magnification that will allow projection of the whole structure onto the detector array will make the image of the particular piece of axon or dendrite much smaller (up to 50 times in our experiments) than the detector and, thus, the signal-to-noise ratio will be degraded. We did not find an intracellular absorption dye that is sensitive enough to membrane potential changes to overcome these difficulties.

Neither of these problems exist in such severe forms in fluorescence measurements, and a dramatic improvement was achieved using cpifluorescence. The factor of crucial interest in this approach, signal-to-noise ratio, has been improved by a factor of roughly 50 over previously available absorption and fluorescence signals from neuronal processes in intact ganglia. It was possible to monitor action potential signals in a $6 \mu \mathrm{m}$ diameter axon with a signal-to-noise ratio of about 30:1 in a single trial utilizing the styryl dye, JPW1114. This improvement depends on (1) the advantages of fluorescence measurements over absorption in recording from small processes, (2) improvements in apparatus to lower the noise and to filter more efficiently; (3) finding a dye that gives relatively large fractional change in fluorescence. The high sensitivity of the styryl dye JPW1114, with the amino-naphthyl moiety, as compared to the two amino-phenyl dyes (RH437 and RH461) is probably based on differences in size and structure of the chromophores (Fromherz and Muller, 1993). However, the precise physical origin of voltage sensitivity is not known. Relatively low signal-to-noise ratio that was recorded with the dye JPW1063, a close analog of JPW1114, is probably related to difficulties in obtaining adequate staining; due to higher hydrophobicity, this dye was difficult to inject and its spread from the point of injection was poor even after $48 \mathrm{hr}$.

Optical recording with good signal-to-noise ratios from neuronal processes has been obtained from neurons in monolayer cultures of dissociated cells (Grinvald et al., 1981, 1982, 1983; Parsons et al., 1991). However, monolayer culture is a low opacity system especially convenient for both absorption and fluorescence measurement. Extending these methods to recording from processes of single neurons in intact invertebrate ganglia has been difficult. Ross and Krauthamer (1984) used transmission measurements to record optical signals from neuronal processes in intact ganglia. The signal-to-noise ratio was not as good as recordings from monolayer culture and extensive averaging (400 trials) was required to obtain signals from processes of single cells using extracellularly applied dye. Furthermore, when many neurons are active in a densely packed neuropile it is a problem to determine the source of the signal if all the cells and processes are stained. Grinvald et al. (1987) 
used intracellularly injected fluorescent dyes to record action potential and synaptic potential signals from individual leech neurons. Due to the relatively low sensitivity of the dyes (fractional change in fluorescence intensity between $0.01 \%$ and $0.1 \%$ ), averaging and bleach correction procedures were important and spatial resolution was sacrificed to improve the signalto-noise ratio. Furthermore, the recording was limited to one location. Because the signal size has been small, it had not been possible to use multisite optical recording to evaluate details of synaptic interactions at the level of neuronal processes. The substantial improvement in signal-to-noise ratio that we report here might facilitate such studies.

Comparison of clectrical and optical signals show that fluorescence changes closely follow the time course of the action potential with some irregular discrepancies at longer times (Fig. 5). We assign these discrepancies primarily to the high-pass filtering of optical signals. Some contributions from the slow noise in optical recording and distortions due to the bleaching of the dye are also possible. With the light intensities we used, the effect of bleaching was small as compared to membrane potential related signals. However, recording slower and longer lasting signals (synaptic potentials) in future experiments may require a bleaching correction (Grinvald et al., 1987). No significant photodynamic damage or pharmacological effects were observed with the dye JPW1114 injected into the cell from a microelectrode filled with a $3 \mathrm{mg} / \mathrm{ml}$ dye solution.

Optical recordings were obtained from cells selectively stained by intracellular injection. Typically, only one neuron in the ganglion was stained and we can safely attribute optical signals to potential transients in specific regions of the neuron. The anatomy of the giant metacerebral cell is such that the cell soma and two main axonal branches are in the same plane of focus for the $10 \times$ objective. Both soma and processes are on or close to the surface so that there was little light scattering. In this situation, it is straightforward to identify the regions from which optical signals originate.

\section{Spike trigger zorte and propagalion}

The location of the spike trigger zone in a neuron is an important functional parameter since it determines the relative weight of different synaptic inputs (e.g., Jacobs and Miller, 1985). When the giant metacerebral cell is stimulated by depolarizing the soma with a current pulse, the site of initiation of the action potential was found to be in the proximal axon. However, this location was variable from cell to cell and might also depend on the stimulus strength, which is difficult to control precisely. Similar results were obtained on barnacle neurons (Ross and Krauthamer, 1984) where the location of the primary initiation zone varied with characteristics of the somatically injected current. In cases where the initiation site of the action potential was in the proximal axonal region adjacent to the soma, we monitored the orthodromic propagation toward the periphery (Fig. 7). In cases where the first spike was initiated in a remote region of an axonal branch, we monitored its antidromic propagation back to the soma (Fig. $8 A, B$ ). The action potential presumably also propagated orthodromically from the initiation site down the axon. However, since signal size declined with distance from the soma, we were not able to reliably record those signals.

One explanation for these observations is that the cell body and proximal axonal regions are less excitable than more distal parts. However, we cannot exclude the possibility that soma and parts of proximal axons adjacent to the cell body are actually nonexcitable and only passively conduct impulses.

There must be at least two separate spike trigger zones in the metacerebral cell in order to account for the recorded pattern of action potential propagation. The functional significance of multiple trigger zones is not clear and remains to be explored, especially during synaptic activation of the neuron.

The location and precise function of the action potential initiation zone in vertebrate neurons is also uncertain. Experiments on Purkinje cells from juvenile rats (Regehr et al., 1992) and neocortical pyramidal neurons (Regehr et al., 1993) provide evidence that the primary spike initiation zone might be in dendrites rather than in the proximal axon region. Contrary to these results, experiments by Stuart and Sakmann (1994) showed that action potentials are initiated first in the axon, as suggested in the original studies on action potential initiation in motor neurons (Coombs et al., 1957), and then actively propagate into the dendritic arbor. Obtaining direct evidence might be facilitated by monitoring membrane potential transients at many sites by using voltage-sensitive dyes and selective staining.

Regional differences in propagation velocity could have important effects on the signal integration at the level of single cells. The change in propagation velocity may reflect a change in the space constant of an axonal segment which, in turn, could determines the pattern of spatial summation of subthreshold signals in the processes. We found that propagation velocity was different in different axonal branches and in different segments of the same branch. Recorded velocities are within the range found previously in molluscan preparations (Pin and Gola, 1984; Stepnoski et al., 1991; Fromherz and Vetter, 1992), but very little is known about how uniform these values are along the neurites of individual cells. Nonuniform propagation has been described previously in the processes of Retzius cells of the leech in culture (Fromherz and Vetter, 1992). The causes of these variability are not known and might be due to differences in both passive and active properties. Also, the functional significance of nonuniform conduction has not been established. Our results show that optical techniques can be used to look for these effects during synaptic activation of the cell.

Figure 9 showed that it is also possible to measure the passive spread of electrotonic responses in the processes. These records were scaled to reflect the relative amplitudes of potential transients at different locations, and the decline of local response amplitude with distance from stimulation site was monitored. It is important that predictions about the space constant of neuronal processes based on optical recordings can now be directly tested by electrical measurements from two locations on the same neuron using patch-electrodes and whole-cell recordings (Stuart and Sakmann, 1994).

\section{Future improvements}

The present results demonstrate adequate sensitivity and tcmporal and spatial resolution to allow monitoring the generation and spread of electrical signals in neuronal processes from anatomically favorable cells, in regions that are as far as $1 \mathrm{~mm}$ from the site of dye injection (cell soma). However, the optical signals are still relatively small and the signal-to-noise ratio declines in amplitude with distance. It is, therefore, important to improve the efficiency of recording.

The most important improvement concerns signal size. Improvement in the fractional change in fluorescence measurements would imply looking for better dyes. Our results suggest 
that more positively charged molecules might give bigger signals. We plan to test additional molecules for better fractional fluorescence changes. The sensitivity of the recording with JPW 1114 would also be improved by increasing the light intensity. The signal-to-noise ratio in optical recording is a complex function of light intensity ( $\mathrm{Wu}$ and Cohen, 1993). At relatively low intensities the limiting noise in the recording is dark, electrical noise found in the system even in the absence of light. In this situation, signal will increase linearly with the light intensity and since dark noise is independent of light, the signal-to-noise ratio will also linearly increase. At intermediate light intensities, the shot noise due to the statistical nature of photon emission and detection becomes the dominant noise in the recording. $\mathrm{Be}$ cause shot noise increases as the square root of light intensity, in the shot noise limited case, the signal-to-noise ratio will also be improved by increasing light intensity, but only proportional to the square root of the intensity increase. At higher light intensities, the fractional shot noise is low and extraneous noise due to vibrations in the light path and movement of the preparation may become dominant. Since both vibrational noise and signal will increase linearly with light intensity, in the vibrational noise limited case, increasing the intensity will not improve the signal-to-noise ratio. In our experiments, the light intensity from somatic regions was roughly 100 times larger than the light levels from distal axonal branches. As a result, the signal-to-noise ratio in recordings from the soma was limited by vibrational noise while the limiting factor in recording from neuronal processes was dark noise. It is evident that the recording from small neuronal processes is far from theoretical and technical limits. By using an arc-lamp in place of tungsten-halogen lamp to increase the excitation light intensity, it should be possible to obtain quickly a significant improvement in signal-to-noise ratio from processes before shot noise becomes apparent, and much before vibrational noise becomes a limiting factor.

Spatial resolution can be further improved by using an array of more elements; these have been recently developed and are in use in several laboratories (Nakashima et al., 1992; Sakai et al., 1993; Vranesic et al., 1993; Wu and Cohen, 1993). Also, higher magnification objectives can be used to image specific parts of neuronal arborization in more detail.

\section{References}

Antić S, Zečević D (1993a) Recording of membrane potential changes from neuronal processes by fluorescence measurements. Soc Neurosci Abstr 19:440.11

Antić S, Zečević D (1993b) Voltage-sensitive dyes: optical recording of membrane potential changes from neuronal processes. 16th ENA Meeting Abstr, p 76.

Bonhoeffer T, Staiger V (1988) Optical recording with single cell resolution from monolayer slice cultures of rat hippocampus. Neurosci Lett 92:259-264.

Borst A, Egelhaaf M (1992) In vivo imaging of calcium accumulation in fly interneurons as elicited by visual motion stimulus. Proc Natl Acad Sci USA 89:4139-4143.

Cleary LJ, Byrne JH (1993) Identification and characterization of a multifunction neuron contributing to defensive arousal in Aplysia. $\mathrm{J}$ Neurophysiol 70: 1767-1776.

Cohen LB, Lesher S (1986) Optical monitoring of membrane potential: methods of multisite optical measurement. Soc Gen Physiol Ser 40: 71-99.

Cohen LB, Wu JY (1990) One neuron, many units. Nature 346:108109.

Coombs BG, Curtis DR, Eccles JC (1957) The interpretation of spike potentials of motoneurones. J Physiol (Lond) 139:198-231.

Egelhaaf M, Borst A, Warzecha A, Flecks S, Wildemann A (1993) Neural circuit tuning fly visual neurons to motion of small objects II.
Input organization of inhibitory circuit elements revealed by electrophysiological and optical recording techniques. J Neurophysiol 69: 340-351.

Fromherz P, Muller CO (1993) Voltage-sensitive fluorescence of amphiphilic dyes in neuron membrane. Biochim Biophys Acta 1150: $111-122$.

Fromherz P, Vetter T (1992) Cable properties of arborized Retzius cells of the leech in culture as probed by a voltage-sensitive dye. Proc Natl Acad Sci USA 89:2041-2045.

Grinvald A, Ross WN, Farber I (1981) Simultaneous optical measurements of electrical activity from multiple sites on processes of cultured neurons. Proc Natl Acad Sci USA 78:3245-3249.

Grinvald A, Hildesheim R, Farber IC, Anglister L (1982) Improved fluorescent probes for the measurement of rapid changes in membrane potential. Biophys J 39:301-308.

Grinvald A, Fine A, Farber IC, Hildesheim R (1983) Fluorescence monitoring of electrical responses from small neurons and their processes. Biophys J 42:195-198.

Grinvald A, Salzberg BM, Lev-Ram V, Hildesheim R (1987) Optical recording of synaptic potentials from processes of single neurons using intracellular potentiometric dyes. Biophys I 51:643-651.

Gupta RK, Salzberg BM, Grinvald A, Cohen LB, Kamino K, Lesher $\mathrm{S}$, Boyle MB, Waggoner AS, Wang CH (1981) Improvements in optical methods for measuring rapid changes in membrane potential. J Membr Biol 58:23-137.

Hodgkin AL, Rushton WAH (1946) The electrical of a crustacean nerve fiber. Proc R Soc Lond [Biol] 133:444-479.

Jacobs GA, Miller JP (1985) Functional properties of individual neuronal branches isolated in situ by laser photoinactivation. Science 228:344-346

Jacobs GA, Miller JP (1988) Analysis of synaptic integration using the laser photoinactivation technique. Experientia 44:361-368.

Kandel ER, Tauc L (1965) Input organization of two symmetrical giant cells in the snail brain. J Physiol (Lond) 183:269-286.

Kim HG, Connors BW (1993) Apical dendrites of the neocortex: correlation between sodium- and calcium-dependent spiking and pyramidal call morphology. J Neurosci 13:5301-5311.

Lasser-Ross N, Miyakawa H, Lev Ram V, Young SR, Ross WN (1991) High time resolution fluorescence imaging with CCD camera. J Neurosci Methods 36:253-262.

Llinas R, Suginori M (1980) Electrophysiological properties of in vitro Purkinje cell dendrites in mammalian cerebellar slices. J Physiol (Lond) 305:197-213.

Loew ML, Cohen LB, Dix J, Fluhler EN, Montana V, Salama G, Young WJ (1992) A naphthyl analog of the aminostyryl pyridinium class of potentiometric membrane dyes shows consistent sensitivity in a variety of tissue, cell and model membrane preparations. J Membr Biol 130:1-10.

London JA, Zečević D, Cohen LB (1987) Simultaneous optical recording of activity from many neurons during feeding in Navanax. $\mathrm{J}$ Neurosci 7:649-661.

Midtgaard J, Lasser-Ross N, Ross WN (1993) Spatial distribution of $\mathrm{Ca}^{2+}$ influx in the turtle Purkinje cell dendrites in vitro: role of transient outward current. J Neurophysiol 70:2455-2469.

Miyakawa H, Lev-Ram V, Lasser-Ross N, Ross WN (1992) Calcium transients evoked by climbing fiber and parallel fiber synaptic inputs in guinea-pig cerebellar neurons. J Neurophysiol 68:1178-1189.

Nakashima M, Yamada S, Shiono S, Maeda M, Satoh F (1992) 448Detector optical recording system: development and application to Aplysia gill-withdrawal reflex. IEEE Trans Biomed Eng 39:26-36.

Obaid AL, Shimizu H, Salzberg BM (1982) Intracellular staining with potentiometric dyes: optical signals from identified leech neurons and their processes. Biol Bull 163:388.

Parsons TD, Salzberg BM, Obaid AL, Raccuia-Behling F, Kleinfeld D (1991) Long-term optical recording of patterns of electrical activity in ensembles of cultured Aplysia neurons. J Neurophysiol 66:316333.

Pin T, Gola M (1984) Axonal mapping of neurosecretory Helix bursting cells. Functional aspects of peripheral multibranched axons. Comp Biochem Physiol 79A:611-620.

Regehr WG, Konnerth A, Armstrong C (1992) Sodium action potentials in the dendrites of cerebellar Purkinje cells. Proc Natl Acad Sci USA 89:5492-5496.

Regehr WG, Kehoe JS, Ascher P, Armstrong C (1993) Synaptically triggered action potentials in dendrites. Neuron 11:145-151. 
Ross WN, Krauthamer V (1984) Optical measurements of potential changes in axons and processes of ncurons of a barnacle ganglion. $\mathrm{J}$ Neurosci 4:659-672.

Ross WN, Werman R (1987) Mapping calcium transients in the dendrites of Purkinje cells from the guinea-pig cerebellum in vitro. J Physiol (Lond) 389:319-336.

Ross WN, Salzberg BM, Cohen LB, Grinvald A, Davila HV, Waggoner AS, Wang CH (1977) Changes in absorption, fluorescence, dichroism, and birefringence in stained giant axons: optical measurement of membrane potential. J Membr Biol 33:141-183.

Ross WN, Miyakawa H, Lev-Ram V, Lasser-Ross N, Lisman J, Jaffe D, Johnston D (1993) Dendritic excitability in CNS neurons: insights from dynamic calcium and sodium imaging in single cells. Jpn J Physiol 43:S83-S89.

Sakai T, Sato K, Momose-Sato Y, Hirota A, Kamino K (1993) Optical multiple-site sectioning of glossopharyngeal response in the early embryonic brainstem. Soc Neurosci Abstr 19:272.2.

Salzberg BM, Obaid AL, Bezanilla F (1993) Microsecond response of a voltage-sensitive merocyanine dye: fast voltage-clamp measurements on squid giant axon. Jpn J Physiol 43:S37-S41.

Segev I, Rapp M, Manor Y, Yarom Y (1992) Analog and digital processing in single nerve cells: dendritic integration and axonal propagation. In: Single neuron computation (McKenna T, Davis J, Zornetzer SF, eds). New York: Academic.
Stepnoski RA, LaPorta A, Raccuia-Behling F, Blonder GE, Slusher RE, Kleinfeld D (1991) Noninvasive detcetion of changes in membrane potential in cultured neurons by light scattering. Proc Natl Acad Sci USA 88:9382-9386.

Stuart GJ, Sakmann B (1994) Active propagation of somatic action potentials into neocortical pyramidal cell dendrites. Nature 367:6972

Tank DW, Sugimori M, Connor JA, Llinas RK (1988) Spatially resolved calcium dynamics of mammalian Purkinje cells in cerebellar slices. Science 242:773-777.

Vranesic I, Iijima T, Ichikawa M, Matsumoto G, Knopfel T (1993) High resolution imaging of electrical signal spread within the granule cell layer of the rat cerebellar slice using a voltage-sensitive dye. Soc Neurosci Abstr 19:652.17.

Wu JY, Cohen LB (1993) Fast multisite optical measurement of membrane potential. In: Fluorescent and luminescent probes for biological activity (Mason WT, ed). New York: Academic.

Xiao C, Zečević D (1988) Optical recording of membrane potential changes from single neurons with intracellular dyes. Biol Bull 175: 319.

Zečević D, Antić S (1992) Absorption voltage-sensitive dyes for intracellular application. Soc Neurosci Abstr 18:857.

Zečević D, Wu JY, Cohen LB, London JA, Hopp HP, Falk XC (1989) Hundreds of neurons in the Aplysia abdominal ganglion are active during the gill-withdrawal reflex. J Neurosci 9:3681-3689. 